\title{
Increased sensitivity of HIV-1 antibody detection
}

\author{
Howard B. URnovitz, Jerrilyn C. STurge \& Toby D. GotTFried \\ Calypte Biomedical, 1440 Fourth Street, Berkeley, California 94710,USA, e-mail: hervdoc@aol.com \\ Correspondence should be addressed to H.B.U.
}

\begin{abstract}
Clinical trial results from 11,344 paired urine and serum samples revealed 1,181 HIV-1-positive individuals confirmed by western blot (WB). There were 25 discrepant samples: 10 were urine enzyme immunassay (EIA) and WB positive, serum non-reactive and serum WB negative or indeterminate, and 15 were serum EIA and WB positive, urine EIA non-reactive or urine WB negative or indeterminate. Serum samples, HIV: 1 antibody WB confirmed, revealed a $99.15 \%$ sensitivity (1,171 out of 1,181); urine samples, HIV-1 antibody WB confirmed, showed a $98.73 \%$ sensitivity $(1,166$ out of 1,181$)$. This study demonstrated that neither serum nor urine results alone are as sensitive for HIV-1 antibody detection as combined results of both samples.
\end{abstract}

Antibody assays provide a convenient and inexpensive method to screen for the detection of HIV-1 infection ${ }^{1}$. Since 1985, tests continue to be licensed in the United States for use with blood products (serum and plasma), oral fluid ${ }^{2}$ and urine ${ }^{3}$. The prevailing concept in HIV-1 diagnostics has been that an HIV-1 antibody response can always be found in the blood except in cases seen during the "window period," when the infection is too early for an antibody response ${ }^{4}$.

Increasing numbers of studies have challenged the concept that blood-based antibodies can always be detected in a non-window period $^{3}$. The detection of the first case of HIV-1 group "O" (ref. 5) showed a urine WB-confirmed antibody presence in a paired serum WB envelope-negative (sero-indeterminate) individual ${ }^{3}$. In a second study, seven EIA seronegative subjects were found to be urine EIA and WB confirmed positive ${ }^{6}$. Follow-up studies on a subset of these individuals demonstrated a blood-related cell-mediated immune response to HIV-1 peptides, suggesting a specialized ${ }^{7}$ or compartmentalized immune response to HIV-1. In a more detailed investigation of 16 HIV-1 discordant couples, it was shown that the HIV1 exposed seronegative partner had a mucosal associated HIV-1 IgA that could be detected in urine or vaginal washes ${ }^{8}$. Finally, three individuals were found to be urine positive and serum EIA non-reactive or WB indeterminate 9 . One EIA-seronegative/urine-positive subject was found to have 46,600 copies of HIV-1 viral RNA/ml plasma.

A clinical study in support of U.S. licensure for the Calypte HIV1 urine EIA was performed on 11,344 individuals to compare the accuracy of HIV-1 urine testing with a U.S. licensed HIV-1 serum test. A total of 10,163 individuals were either EIA non-reactive or WB negative or indeterminate for HIV-1 antibodies with both serum and urine samples. 1,156 individuals were WB-confirmed positive by both serum and urine samples. However, there were 25 discordant samples: 10 were urine $\mathrm{WB}$-confirmed positive, while the paired serum samples were EIA non-reactive and WB negative or indeterminate, and 15 were serum $\mathrm{WB}$ positive, while the paired urine samples were EIA non-reactive or WB negative or indeterminate. The serum test detected $99.15 \%(1,171$ out of 1,181$)$ of the HIV-1 antibody-positive individuals. The urine test detected $98.73 \%(1,166$ out of 1,181 ) of the HIV-1 antibody-positive individuals. If the criteria for HIV-1 antibody positivity were that at least one sample (serum or urine) be WB-confirmed positive, the combined results would have detected $100 \%(1,181$ out of 1,181$)$ of individuals in this study.

Like most other chronic diseases, AIDS is a complicated multifactorial, multistep process, with HIV-1 infection being a principal component ${ }^{3}$. Accurate diagnosis of HIV-1 infection is important in determining an individual's risk for developing AIDS. Accuracy is complicated by false-positive and false-negative results.

We have reported on the contribution of endogenous retroviral genes $^{10}$ to false positivity ${ }^{3}$. With regard to false-negative reactions, it would appear that in some limited infections, a compartmentalized response occurs in which expression of HIV-1 or its respective immune response is limited to a restricted number of organs and tissues. The results of this large clinical study support this concept. Public health policies may need to be revised in settings where absolute sensitivity for HIV-1 antibody testing reflects life-or-death decisions. Combination sampling with at least two body fluids may assist in the process of such decision-making. In addition, the use of combined sampling may permit easier detection of populations with "discordant" results. Study of individuals with discordant results may lead to further discoveries of the body's natural mechanisms in combating HIV-1 infection?

\section{Methods}

A total of 11,344 paired urine and serum samples were collected at 11 sites in the United States and Haiti from subjects at low and high risk of infection with HIV-1. The samples were tested at seven sites. To ensure accuracy in sample handling and testing, sites were selected on the basis of experience with clinical trials. Undiluted urine specimens were tested in an EIA with an HIV-1 recombinant envelope protein". Repeatedly reactive specimens were tested with a modification of a licensed WB procedure ${ }^{3}$. Serum specimens were tested by licensed EIA, and repeatedly reactive specimens were tested by WB. In most cases, discordant samples were retested by EIA and WB.

\section{RECEIVED 3 SEPTEMBER; ACCEPTED 26 SEPTEMBER 1997}

1. Gottried, T. D. \& Urnovitz, H.B. HIV-1 Testing: Product Development Strategies. Trends in Biotechnology. 8, 35-40 (1990).

2. Gallo, D., George, J.R., Fitchen, J.H., Goldstein, A.S. \& Hindahl, M.S. Evaluation of a sytstem using oral mucosal transudate for HIV-1 antibody screening and confirmatory testing. JAMA 277, 254-8 (1997).

3. Urnovitz, H. B., W. H. Murphy, T. D. Gottfried \& Friedman-Kien, A. E. Urine-Based Diagnostic Technologies. Trends in Biotechnology 14, 361-364 (1996).

4. Hashida S. et al. Shortening of the window period in diagnosis of HIV-1 infection by simultaneous detection of $\mathrm{p} 24$ antigen and antibody $\operatorname{lgG}$ to $\mathrm{p} 17$ and reverse transcriptase in serum with ultrasensitive enzyme immunoassay. / Virol Methods 62, 43-53 (1996).

5. Charneau P. et al. Isolation and envelope sequence of a highly divergent HIV-1 isolate: definition of a new HIV-1 group. Virology 205, 247-53 (1994).

6. Urnovitz, H. B. et al. HIV-1 antibody serum negativity with urine positivity. Lancet 342 , 1458-1459 (1993).

7. Shearer, G. M. \& Clerici, M. Protective immunity against HIV infection: has nature done the experiment for us? Immunol. Today 17, 21-24 (1996).

8. Mazzoli, $S$ et al. HIV-Specific Mucosal and Cellular Immunity in HIV-Seronegative Partners of HIV-Seropositive Individuals. Nature Medicine, 1250 (in Press, 1997).

9. Sordillo, E.M. et al. Western Blot Testing of HIV EIA Reactive Urine Samples. Am. Soc. Micro. Abstr. \#V-70 (1997).

10. Umovitz, H. B. \& Murphy, W.H. Hurnan Endogenous Retroviruses: Nature, Occurrence and Clinical Implications in Human Disease. Clin Micro Reviews 9, 72-99 (1996).

11. Calypte ${ }^{\mathrm{TM}}$ HIV-1 Urine EIA Package Insert, Berkeley, CA., Calypte Biomedical (1996). 